\title{
A Photon Veto Detector for the CKM Experiment
}

\author{
Erik Ramberg, Peter Cooper, and Robert Tschirhart
}

\begin{abstract}
Fermilab experiment E921, or Charged Kaons at the Main Injector (CKM), will measure the branching ratio of the ultrarare kaon decay: $\mathrm{K}^{+} \rightarrow \pi^{+} \nu \nu$. To do this, CKM will need to reject the far more copious decay $\mathbf{K}^{+} \rightarrow \pi^{+} \pi^{0} \rightarrow \pi^{+} \gamma \gamma$ by a factor of more than 10 billion. Much of this rejection will come from the detection of either or both of the photons by our photon veto system. The main CKM photon veto system is based on 80-layer sandwiches of 1-mm $\mathrm{Pb}$ and 5-mm fast scintillator with wavelength shifting fibers inside a vacuum decay volume, read out with photomultiplier tubes outside the vacuum. We describe the design of the CKM photon veto system and the testing of a prototype in an electron beam at Jefferson National Laboratory, demonstrating an order of magnitude better performance than the required $3 \times 10^{-5}$ inefficiency for a $1 \mathrm{GeV}$ electromagnetic shower.
\end{abstract}

Index Terms-Kaon decay experiments, particle detectors, photon veto detectors.

\section{INTRODUCTION}

$\mathbf{E}$ XPERIMENT E921 at Fermilab, known as "Charged Kaons at the Main Injector" (CKM) will measure the branching ratio of the ultrarare charged kaon decay $\mathrm{K}^{+} \rightarrow \pi^{+} \nu \nu$ to a precision of $10 \%$. This measurement can be used to calculate a value for the Cabibbo-Kobayashi-Maskawa quark mixing matrix element $\left|\mathrm{V}_{\mathrm{td}}\right|$ and, by comparing to measurements in the B meson system, potentially the Standard Model of particle physics. The Standard Model predicts the branching ratio of this mode to be on the order of $0.8 \times 10^{-10_{1}}$ . The only measurement of this decay is by the E787/E949 experiment at Brookhaven National Laboratory. This group has seen three of these decays, equivalent to a branching ratio of about $1.5(+1.3 /-0.9) \times 10^{-10}[1]$. One of the most common decay modes of the kaon, " $\mathrm{K} \pi 2$ " or $\mathrm{K}^{+} \rightarrow \pi^{+} \pi^{0} \rightarrow \pi^{+} \gamma \gamma$, has a branching ratio of 0.21 . Given the smaller acceptance for our $\mathrm{K} \pi \nu \nu$ signal than for the $\mathrm{K} \pi 2$ background, and if we wish to keep this background at a level well below the signal, we need approximately 12 orders of magnitude event rejection. We calculate that kinematic rejection will reduce the $K \pi 2$ background mode by only $10^{5}$. The rest of the necessary $10^{7} \mathrm{~K} \pi 2$ rejection must come from detection of the final state photons. For that reason, we have designed a robust and powerful set of photon veto detectors within the apparatus. This paper describes the design of the dominant photon veto detector in CKM, the Vacuum Veto System (VVS), and a measurement of its inefficiency for electrons of $1 \mathrm{GeV}$.

Manuscript received April 12, 2004; revised May 5, 2004. This work was supported by the U.S. Department of Energy.

The authors are with the Fermi National Accelerator Laboratory in Batavia, Illinois, 60510 USA.

Digital Object Identifier 10.1109/TNS.2004.836738

${ }^{1}$ See A. Buras' lectures in preprint hep-ph/0101336.

\section{DESCRIPTION OF THE CKM EXPERIMENT}

The layout of the CKM apparatus is shown in Fig. 1. The spectrometer is designed to redundantly measure the parameters of the incoming $\mathrm{K}^{+}$and the outgoing $\pi^{+}$and to veto all other particles. The $\mathrm{K}^{+}$beam is measured by three sets of detectors: a set of upstream wire chambers; a beam Ring Imaging Cerenkov counter (RICH); and a detector to measure the kaon trajectory before its entrance into the vacuum volume. There is a long vacuum decay volume, composed of many VVS modules, after which the final state $\pi^{+}$is measured by a set of straw tube drift chambers and a RICH. A lead/scintillator detector exists at the entrance of the vacuum decay volume to veto any beam interactions in the upstream vacuum window. There is a CsI calorimeter after the $\pi^{+} \mathrm{RICH}$ to act as a forward photon veto system (FVS). Behind the FVS is a muon veto system and a small lead/scintillator veto covering the beam hole region. A comprehensive description of CKM and each of these detectors, and our physics motivation, can be found at our web page: http://www.fnal.gov/projects/ckm/Welcome.html2 .

\section{DESCRIPTION OF THE CKM VACUUM VETO SySTEM}

As outlined above, there are several photon veto systems in the CKM apparatus. The required performance is most stringent for the largest system, the VVS, which intercepts approximately $90 \%$ of the photons from $\mathrm{K} \pi 2$ decays. The inefficiency requirements for the VVS as a function of photon energy are shown in Fig. 2. Our simulations of the experiment show that background from $\mathrm{K} \pi 2$ decays will likely be on the order of $4 \%$ of our signal given this performance of the veto system. If the experiment can achieve better inefficiency performance in the VVS, then the background from $\mathrm{K} \pi 2$ decays will be correspondingly smaller. All of the high-energy photons arising from decays are at small angles with respect to the beam direction. Our design thus concentrates on detection in the forward direction and consists of 1-m-long modules, with alternating layers of lead and scintillator, oriented in vertical annular rings around the beam. We have chosen a 1-mm lead/5-mm scintillator geometry based on the success of the BNL experiment E787 in using this kind of detector for their measurement of the $\mathrm{K} \pi \nu \nu$ decay.

Low energy photons arising from $\mathrm{K} \pi 2$ decay have a very high inherent inefficiency of detection due to electromagnetic shower fluctuations in the lead/scintillator sandwich. Also, low energy photons are not present in every $\mathrm{K} \pi 2$ decay and, if present, are invariably at high angles with respect to the beam direction. Since these high angle photons have a large inherent detection inefficiency, it is reasonable to envision not hermetically covering the full vacuum decay region with photon vetoes. We

\footnotetext{
${ }^{2}$ See especially the CKM proposal to the Fermilab PAC, where the physics and measurement technique of the experiment are described in detail.
} 


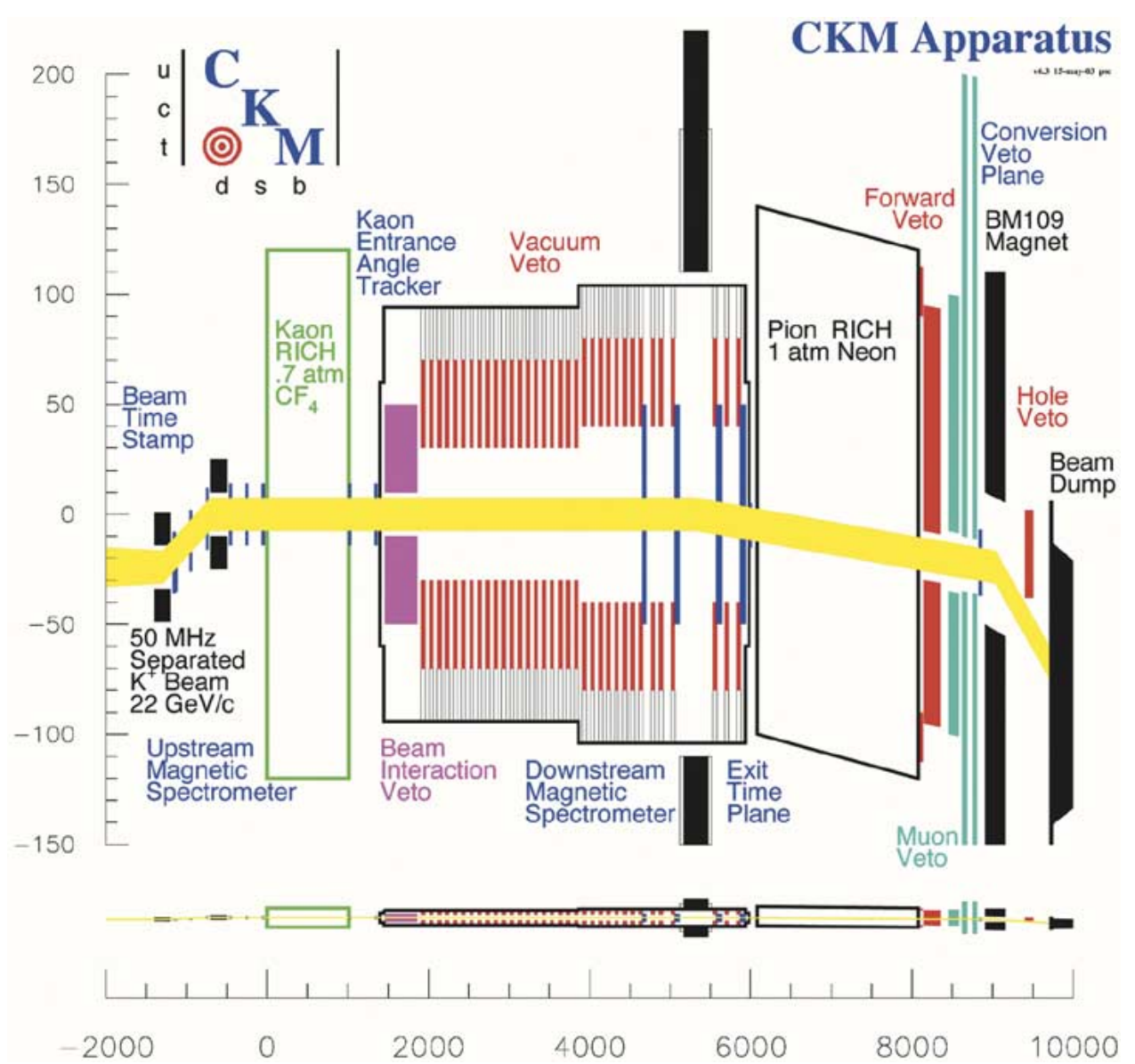

Fig. 1. Layout of the Fermilab CKM experiment. Note the expanded transverse scale. The lower figure shows the experiment in true scale.

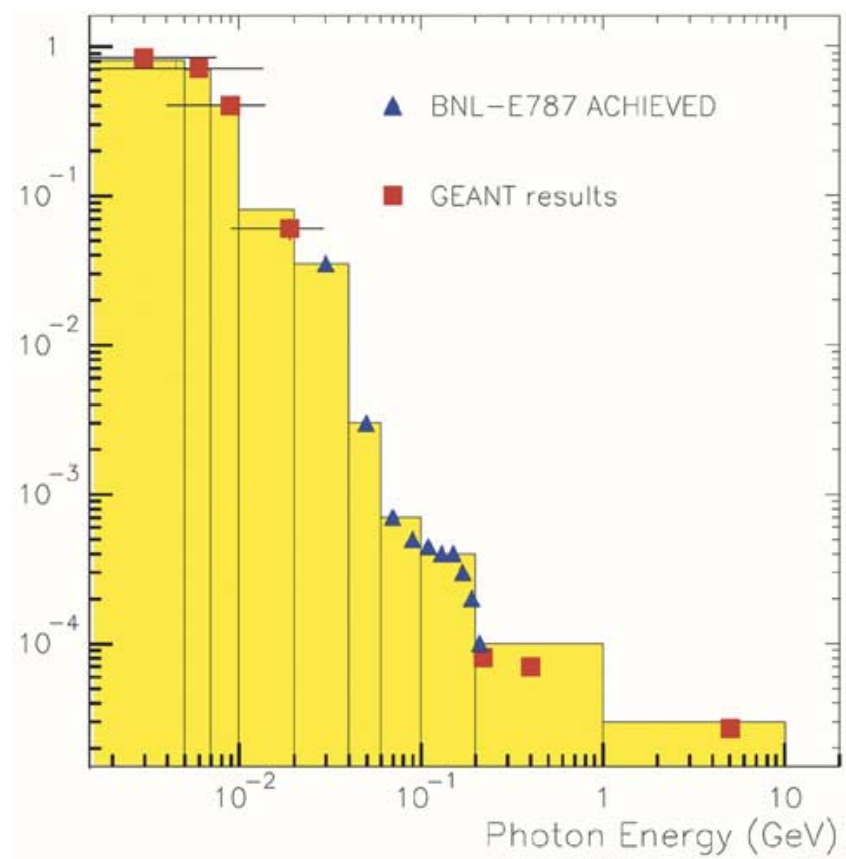

Fig. 2. The required photon detection inefficiency in the VVS detector. We have indicated photon inefficiencies in the BNL E787 experiment, which uses a similar geometry for their central photon veto detector. We have also indicated results for high- and low-energy inefficiencies from a GEANT simulation of the detector.

have determined that a $50 \%$ coverage along the decay region will have only a modest effect on our total $\mathrm{K} \pi 2$ detection in- efficiency while saving a great deal on construction costs. We have, therefore, chosen a scheme where the vacuum decay region is composed of 1-meter vacuum modules, with the annular lead/scintillator sandwich filling half of each module. There are two sizes for this module, with the smaller one having a $30-\mathrm{cm}$ inner aperture radius, and the active detector extending out to a radius of $70 \mathrm{~cm}$. The larger size has a $40-\mathrm{cm}$ inner aperture radius, extending out to a $80-\mathrm{cm}$ outer active radius. There are 20 of the small modules and 14 of the larger modules in the complete VVS detector. The complete active area of the detector is shown in Fig. 1. We have recently completed an engineering design for the VVS module. A solid model of the design is shown in Fig. 3.

To ensure a high veto efficiency, it is important to emphasize redundancy in shower detection and scintillation light readout. Our preliminary design for the scintillator tiles that make up the active part of the detector is a trapezoidal shape with wavelength shifting fibers leading to a photomultiplier. There are 16 of these tiles that make up a complete annular layer. We cannot allow any cracks in photon shower readout and so we will stagger every other layer of scintillator by half a tile in the phi direction. To establish redundancy, odd and even layers of scintillator are read out by a separate photomultiplier. The stack of lead and scintillator is supported at the midpoint by a steel sheet, replacing one of the lead sheets, which attaches to the body of the vessel. The scintillator tiles on either side of this midpoint are read out by separate photomultipliers, leading to segmentation in the beam direction. Each module thus has 64 photomultipliers. 


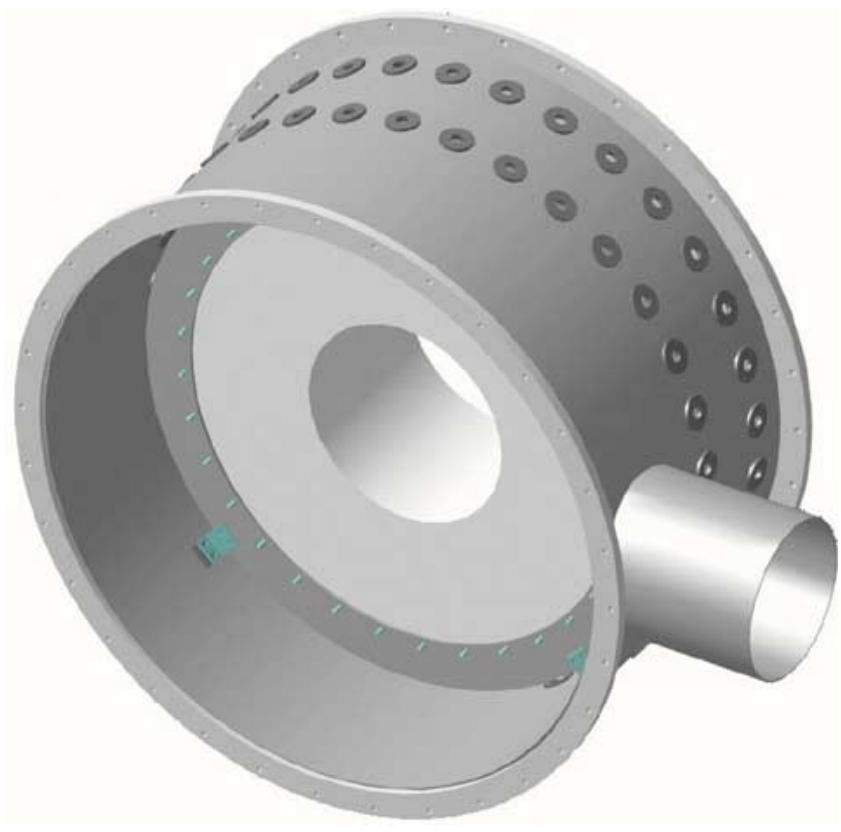

Fig. 3. Solid model rendering of the engineering design of a small vacuum veto module.

One of the most difficult aspects of the VVS detector is that it must contain a vacuum throughout its 40-m extent. Beam gas interactions are a potentially significant source of background events. We have determined that a pressure of $1 \mu$ torr must be achieved to reduce this background to a manageable level. The inner surfaces of each module are painted with a low outgassing paint. The fibers from the scintillator tiles lead to a vacuum sealed polystyrene window fixture mounted on the VVS vessel wall. The photomultipliers are mounted outside the vacuum vessel.

\section{Construction of a VVS Module Prototype}

To understand the construction issues with the VVS design and to test its properties, a prototype of a VVS module was built with only 2 of the 16 phi sectors and the full 80 layers of scintillator and lead. This prototype was housed in a vacuum vessel with one curved wall to simulate the cylindrical VVS module. We used cast Bicron 404 scintillator cut into trapezoidal shapes for our tiles. Sixteen radial grooves were machined in the scintillator, eight on each side of the tile, and Bicron BCF92 multiclad WLS fiber were tack glued into place. The fiber ends were polished and aluminized before installation to enhance their light collection efficiency. The fibers for each tile led to a ferrule where they were glued into place and polished. Thus, each tile could be independently tested and then installed in the module. There are 20 tiles and ferrules that lead to each photomultiplier window. The lead sheets spanned both phi sections and were produced by stamping and subsequent flattening by a press. The tiles and lead sheets were alternately placed onto machined rods that held the end of the tiles in place and a ring segment clamped the stack at the top. A sheet of Tyvek was placed on either side of each scintillator layer. The inner edges of the tiles were bound

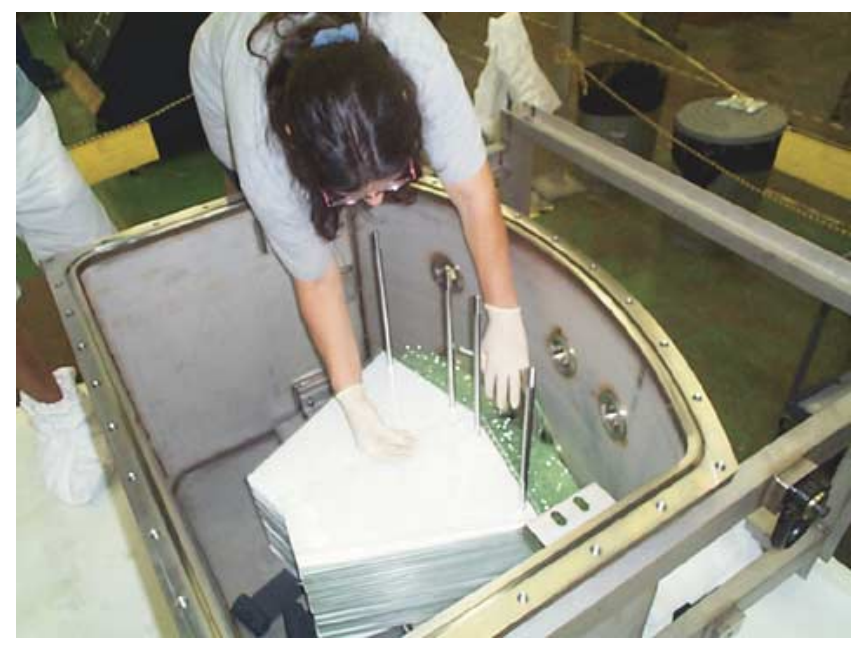

Fig. 4. Photograph of the VVS prototype as the tiles are being placed onto the stack.

only very loosely by a thin Tedlar strip. Alternate layers of scintillator were shifted in phi only by $1 \mathrm{~cm}$ in the prototype. Fig. 4 shows a picture of the prototype module during construction.

The prototype operates as a vacuum vessel and we tested it extensively for outgassing. We determined that the vessel filled with 160 scintillator tiles, their fibers, Tyvek sheets, and lead sheets did not have a significant outgassing rate. Extrapolated to the full VVS detector, we estimate an initial outgassing rate of at most 9,000 L/s at $1 \mu$ torr. The veto prototype photomultiplier tubes (Electron Tubes 9954KB) were instrumented with a base that consisted of a conventional high-voltage divider and two output signal paths. The mean supply high voltage was set to $-1350 \mathrm{~V}$, corresponding to a gain of $5 \times 10^{5}$. One of the output signal paths connected the photomultiplier anode to a gated charge integrator (LRS-2249A ADC). The ADC digitized the anode pulse with a sensitivity of $250 \mathrm{fC} /$ count, where $1 \mathrm{MeV}$ of energy deposited in the plastic scintillator corresponded to about 5 ADC counts. The second output signal path connected the last dynode of the photomultiplier to an on-base discriminator circuit that fired at a voltage of $4 \mathrm{mV}$, corresponding to a energy deposit of $1 \mathrm{MeV}$ in the plastic scintillator. The peak-to-peak electronic noise of the on-base discriminator circuit was $0.5 \mathrm{mV}$. The on-base discriminator drove a low-voltage digital signal (LVDS) signal off-base to a module which converted the LVDS signal to a ECL signal which was subsequently digitized with a LRS-3377 TDC. The TDC measured the dynode pulse time with respect to the event trigger time with a sensitivity of $1 \mathrm{~ns} /$ count.

\section{Test of the VVS PRototype in AN Electron BeAm}

Because the veto inefficiency for high-energy photons is so crucial to the CKM experiment, it was important to test the VVS prototype with real beam. We determined that there was no existing facility that can deliver a known high-energy photon beam with tagging efficiency of better than $10^{-5}$. Conversely, it is possible to tag electrons at this level. Thus, we chose to measure inefficiencies of electron detection.

We installed the prototype in a tertiary electron beam in Hall $B$ at Jefferson Laboratory in Virginia. The beamline delivers 5 $\mathrm{GeV}$ electrons at $50 \mathrm{nA}$. These electrons impinge on a $3 \times 10^{-4}$ 


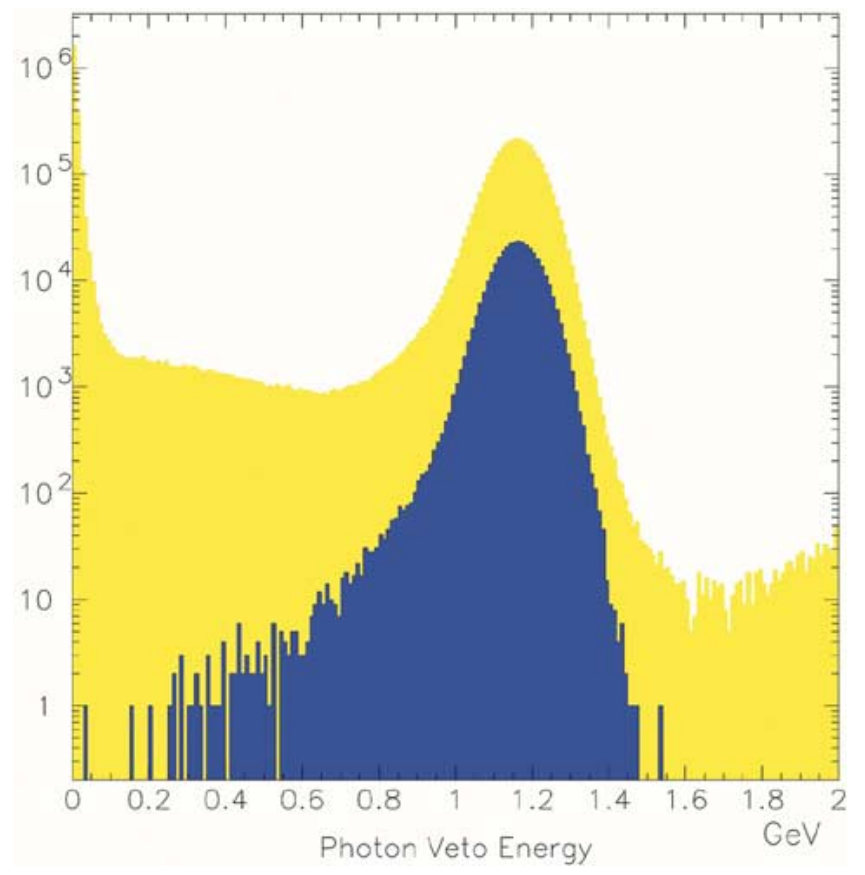

Fig. 5. Detector summed energy for all triggered events (yellow) and for events that pass strict trajectory cuts (blue).

radiation length foil to produce a photon beam. The scattered incident electron is tagged by a magnet and hodoscope array, allowing the photon to proceed down the beamline. The photon beam hits a $1 \times 10^{-3}$ radiation length foil converter, which produces an electron-positron pair a small fraction of the time. This tertiary production produced electron rates that were manageable for inefficiency measurements. A second spectrometer magnet bent these particles into a double arm hodoscope array. Our VVS prototype detector was situated behind a lead wall after one of the arms. The wall had a square hole in it to allow some fraction of the electrons to pass through to our detector. Veto scintillators were placed around the edges of this hole. The beam was centered on a point 1 in in from the edge of the tile stack and on the line between the two phi sections of our prototype.

The trigger electronics and Data Acquisition systems were built with conventional NIM and CAMAC modules interfaced to a PC running Linux. The entire system operated, stand-alone, in Hall B at Jefferson Lab. All operations were done via remote login over the Internet with data logged on a local disk and later copied back to Fermilab. About $30 \times 10^{6}$ events, totaling 7.6 $\mathrm{Gb}$, were taken by triggering on a coincidence of horizontal (H) and remotely moveable vertical (F) trigger counters. Signals from all the trigger counters were discriminated and digitized with LRS 3377-TDCs. The data recorded for each event included the leading and trailing edge times for every signal from each counter in a 512-ns time window centered around the trigger time. Online analysis of these data allowed the selection of event samples where both the electron and positron were cleanly tagged with no additional activity at or near the time of the trigger in the vetoes and other counters.

A full Monte Carlo simulation was made of the Jefferson Lab setup. It predicted mechanisms where the incoming electron would lose most of its energy upstream before hitting the detector and thus mimic an inefficiency. Careful selection of events allowed us to screen out these events. We demanded that the electron have a strict trajectory from the center of the converter to the center of the trigger region. No energy was allowed in the veto counters surrounding the hole in the lead wall. The positron was allowed to have a wide range of trajectories. By changing the field strength of the spectrometer magnet, we studied the prototype response to $0.5,0.8$, and $1.2 \mathrm{GeV}$ electrons. The result for the detector's summed energy for the $1.2 \mathrm{GeV}$ data is shown in Fig. 5 for all triggered events and for events that satisfy our strict trajectory requirements. Out of 354083 events that satisfy our cuts, there is only one event below $150 \mathrm{MeV}$ of detected energy. There were no events in which the discriminated dynode signal, leading to the TDC, did not fire.

A very conservative threshold to calculate inefficiency of the detector is $80 \mathrm{MeV}$, which is equivalent to one minimum ionizing particle's energy deposit. For that threshold, our data translates to an inefficiency of approximately $3 \times 10^{-6}$, an order of magnitude better than our requirement for the CKM experiment. Our thresholds in the experiment will be considerably lower than $80 \mathrm{MeV}$, on the order of $1-5 \mathrm{MeV}$, and we anticipate a very high performance for the CKM vacuum veto system detectors.

\section{SUMMARY}

In summary, we have established a design for the predominant photon veto detector in the CKM experiment at Fermilab. This vacuum veto system consists of modular 1-m detectors, each one $50 \%$ filled with annular rings of alternating 1-mm lead and 5-mm scintillator, read out by wavelength shifting fiber into photomultipliers. The design has significant redundancy in the readout of the detector.

We have built a $1 / 8$ section of one of these modules and installed it in a $1.2 \mathrm{GeV}$ test beam of electrons at Jefferson National Laboratory. By demanding a strict trajectory of the electrons in the test beam spectrometer, we were able to discard events where the electron had lost most of its energy in one of the two upstream hodoscope arrays. These events would have mimicked an inherent inefficiency. Using a very conservative threshold on the energy measurement we have demonstrated a $3 \times 10^{-6}$ inefficiency of our detector to $1.2 \mathrm{GeV}$ electrons. If this same inefficiency holds for photon detection, then the predicted main background for CKM will be significantly reduced from our requirements.

\section{ACKNOWLEDGMENT}

The authors greatly thank the staff of Jefferson National Laboratory and the Hall B personnel for their kind and capable assistance in our measurements there.

\section{REFERENCES}

[1] V. V. Anisimovsky, A. V. Artomonov, B. Bassalleck, B. Bhuyan, E. W. Blackmore, and D. A. Bryman et al., "Improved measurement of the $\mathrm{K}^{+} \rightarrow \pi^{+} \nu \nu$ branching ratio," Phys. Rev. Lett., vol. 93, 2004. 